\title{
Cell adhesion to protein-micropatterned-supported lipid bilayer membranes
}

\author{
Lance Kam, Steven G. Boxer \\ Department of Chemistry, Stanford University, Stanford, California 94305-5080
}

Received 12 June 2000; revised 14 November 2000; accepted 16 November 2000

\begin{abstract}
A new method for constructing controlled interfaces between cells and synthetic supported lipid bilayer membranes is reported. Microcontact printing is used to define squares and grid lines of fibronectin onto glass, which subsequently direct the self-assembly of fluid lipid bilayers onto the complementary, uncoated regions of the surface. Features of fibronectin as small as $5 \mu \mathrm{m}$ effectively control the lateral organization of the lipid bilayers. These fibronectin barriers also facilitate the adhesion of endothelial cells, which exhibit minimal adhesion to fluid supported lipid bilayers alone. Cells selectively adhere to the features of fibronectin, spanning over and exposing the cells to the intervening regions of supported lipid bilayer. Cell spreading is correlated with both the geometry and dimensions of the fibronectin barriers. Importantly, lipids underlying adherent
\end{abstract}

cells are laterally mobile, suggesting that, in contrast to the regions of fibronectin, cells were not in direct contact with the supported membrane. Protein micropatterning thus provides a valuable tool for controlling supported membranes and for juxtaposing anchorage-dependent cells with lipid bilayers. These systems should be generally useful for studying specific interactions between cells and biomolecules incorporated into supported membranes, and as an approach for integrating living cells with synthetic, laterally complex surfaces. (c) 2001 John Wiley \& Sons, Inc. J Biomed Mater Res 55: 487-495, 2001

Key words: micropatterning; microcontact printing of proteins; supported lipid bilayers; cell adhesion; endothelial cells

\section{INTRODUCTION}

Cell-cell communication is mediated in large part by membrane-associated proteins. Substratesupported lipid bilayers offer a unique system with which to study the interaction of cells with membrane proteins, and to potentially utilize these biomolecules to modulate and investigate cellular function. Supported lipid bilayers consist of two leaflets of phospholipids in close association with a hydrophilic surface such as glass. A thin layer of water several nanometers thick separates the membrane from the support. ${ }^{1-3}$ Consequently, molecular components in supported lipid bilayers of appropriate composition freely diffuse within the plane of the membrane, mimicking a property of cellular membranes that is essential for many cellular functions. ${ }^{4-6}$ Furthermore, the composition and fluid properties of supported lipid

Correspondence to: S.G. Boxer

Contract grant sponsor: NIH Genome Training Grant (LK) Contract grant sponsor: NSF Biophysics Program

Contract grant sponsor: MRSEC Program of the NSF; Contract grant number: DMR-9808677

(c) 2001 John Wiley \& Sons, Inc. bilayers are easily controlled, providing a robust tool for the study of systems, ranging from membraneassociated biomolecules (e.g., integrins, gap junctions, ion channels, GPI-anchored proteins, and synthetic peptides) to cells of the immune system. ${ }^{7-11}$

Interfacing anchorage-dependent cells such as hepatocytes, endothelial cells, and neurons with synthetic surfaces using lipid bilayers may also be useful in bioengineering applications, including biomaterial design, cell isolation, and high throughput screening of small molecules that modulate cell-membrane interactions. However, anchorage-dependent cells exhibit minimal adhesion onto fluid phospholipid structures; both the fluidity of lipid bilayers and the ability of specific lipids to resist protein adsorption contribute to the inability of cells to form stable attachments to supported membranes. ${ }^{12}$ In this report, we explore the use of recently developed micropatterning techniques to facilitate adhesion of anchorage-dependent cells onto surfaces containing supported lipid bilayers. Previously, our laboratory has described the microfabrication of barriers that direct and corral lipid diffusion on surfaces by using materials such as $\mathrm{TiO}_{x}$ and photoresist, ${ }^{13,14}$ or by selectively removing regions of the assembled bilayer through either scratching ${ }^{15,16}$ or blotting. ${ }^{17}$ Our laboratory has also demonstrated that 
the protein bovine serum albumin, patterned onto substrates by microcontact printing, ${ }^{18-21}$ can be used as a barrier material. ${ }^{22}$ This development suggested that by creating barriers of biologically active proteins, it may be possible to introduce added functionality to micropatterned lipid bilayers. As outlined schematically in Figure 1(A), we micropatterned supported lipid bilayer membranes using the cell adhesive protein fibronectin. These protein barriers not only pattern and corral the supported bilayers, but also provide stable anchorages for cells, thereby promoting and directing the interaction between adherent cells and supported membranes.

\section{MATERIALS AND METHODS}

\section{Substrate modification}

Vesicle preparation

Stock solutions of small unilamellar vesicles (SUV) were prepared by extrusion using standard techniques. Briefly, egg phosphatidylcholine (egg PC; Avanti Polar Lipids, Alabaster, AL) was dried from chloroform in glass flasks, then desiccated under vacuum for at least $90 \mathrm{~min}$. These lipids were reconstituted in deionized water at a concentration of $5 \mathrm{mg} / \mathrm{mL}$, then extruded through 50-nm pore size polycarbonate membranes (Avanti) using a LiposoFast unit (Avestin, Inc., Ottowa, ON, Canada). For visualization of lipid bilayers, vesicles of egg PC were prepared with either $1 \mathrm{~mol}$ $\%$ of a negatively charged, Texas $\operatorname{Red}^{\circledR}$-labeled phosphatidylethanolamine (TR-PE; Texas Red ${ }^{\circledR}$ 1,2-dihexadecanoylsn-glycero-3-phosphoethanolamine; Molecular Probes, Eugene, OR), or $2 \mathrm{~mol} \%$ of a neutral, NBD-labeled phosphatidylethanolamine (NBD-PE; 1-palmitoyl-2-[12-[(7-nitro2-1,3-benzoxadiazol-4-yl)amino]dodecanoyl]-sn-glycero-3phosphoethanolamine; Avanti). The inclusion of either of these fluorescently labeled lipids into bilayers of egg PC did not influence subsequent cellular response.

\section{Surface micropatterning}

Protein-micropatterned lipid bilayer surfaces were prepared as outlined in Figure 1(A). Borosilicate glass coverslips (VWR Scientific, Media, PA) were cleaned by immersion into Linbro 7X detergent (ICN Biomedicals, Inc., Aurora, $\mathrm{OH})$, diluted $1: 3(\mathrm{v} / \mathrm{v})$ in deionized water, baked at $450^{\circ} \mathrm{C}$ for $4 \mathrm{~h}$, and then micropatterned with fibronectin by microcontact printing. ${ }^{18,20-22}$ Polydimethylsiloxane (PDMS; Sylgard 184; Dow Corning, Midland, MI) elastomer stamps containing topological representations of a microscale pattern, detailed in the section entitled "Micropattern Geometry," were oxidized for $20 \mathrm{~s}$ in air plasma using a cleaning/
A Stamp fibronectin
onto glass
substrate

Incubate with lipid vesicles

Substrate with patterned protein and lipid bilayer

Incubate with serum albumin

Cell adhesion onto micropatterned surface
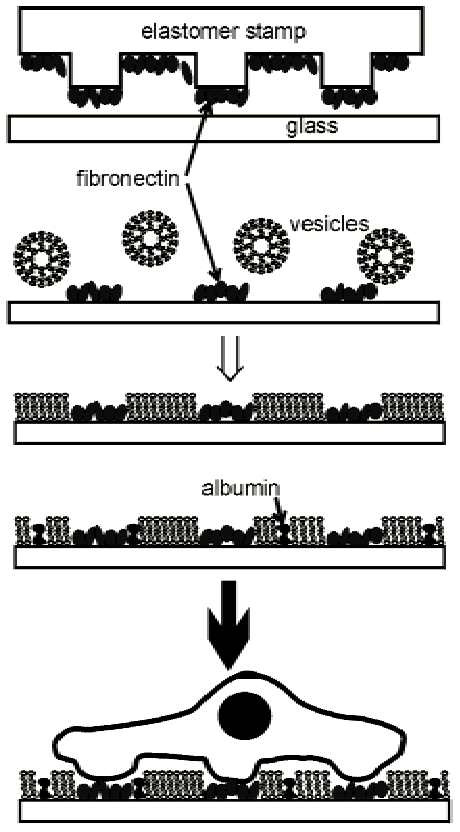

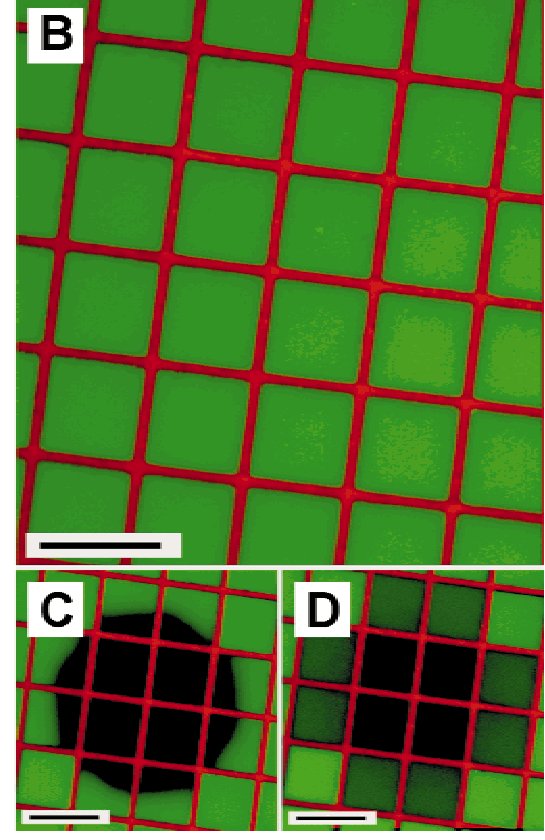

Figure 1. Micropatterning of substrates with fibronectin and phospholipid bilayers. (A) Schematic outlining the microcontact printing process used to create fibronectin barriers on a supported lipid bilayer surface. (B) A surface containing gridlines of fibronectin measuring $5 \mu \mathrm{m}$ in width and spaced $40 \mu \mathrm{m}$ apart, shown here as light gray lines (and in red in the online, color version), surrounding corrals of supported lipid bilayers that contain a fluorescently labeled lipid, NBD-PE, and appear as an array of dark gray (green) squares. (C) An octagonal pattern was photobleached onto an array of 16 lipid corrals. This image was taken immediately after photobleaching. Different fractions of NBD-PE in adjacent corrals underwent photodamage. (D) The same region shown in panel (C), 10 min after photobleaching. Lipids within each corral mixed completely, demonstrating both that the lipid bilayers were fluid and that neighboring corrals were isolated from each other. The scale bar in each image $=50 \mu \mathrm{m}$. [Color figure can be viewed in the online issue, which is available at www.interscience.wiley.com.] 
sterilization unit (Harrick Scientific Corp., Ossining, NY), and were then coated with $100 \mu \mathrm{g} / \mathrm{mL}$ of fibronectin (Sigma, St. Louis, MO) in $0.01 \mathrm{M}$ phosphate buffer ( $\mathrm{pH} 7.3$ ) for 15 min. The stamps were dried using nitrogen gas, and then placed in contact with a coverslip for $15 \mathrm{~min}$; for the duration of this period, a $40 \mathrm{~g}$ weight was placed on each $1 \times 1$ $\mathrm{cm}^{2}$ stamp. After separation from the stamps, the micropatterned coverslips were rinsed in phosphate buffer $(\mathrm{PB} ; 0.01$ $M$ phosphate, $140 \mathrm{mM} \mathrm{NaCl}, \mathrm{pH} 7.3$ ), rinsed in water, and then dried using nitrogen.

Lipid bilayers were formed on these patterned surfaces by vesicle fusion. ${ }^{7,23}$ Stock solutions containing SUVs of either egg PC, egg PC supplemented with 1 mol \% TR-PE (egg PC/TR-PE), or egg PC supplemented with 2 mol \% NBD-PE (egg PC/NBD-PE) were diluted 1:3 in PB, then placed in contact with the micropatterned surface of each coverslip for $30 \mathrm{~s}$. Without exposing this surface to air, the coverslips were rinsed with $P B$, incubated with a solution of $10 \mu \mathrm{g} / \mathrm{mL}$ of fatty-acid-free bovine serum albumin (BSA; Boehringer Mannheim Biochemicals, Indianapolis, IN) in PB at room temperature for $1 \mathrm{~h}$, then rinsed with $\mathrm{PB}$.

Unpatterned, reference substrates were prepared using similar techniques. Clean coverslips were incubated with SUVs of egg PC (stock solutions diluted 1:3 in PB), and then rinsed extensively with $\mathrm{PB}$. As specified, some of these coverslips were subsequently incubated with $10 \mathrm{mg} / \mathrm{mL}$ of BSA in $\mathrm{PB}$ for $1 \mathrm{~h}$, then rinsed with $\mathrm{PB}$.

In preparation for cell experiments, substrates were attached to the bottom of wells of 6-well tissue culture plates using vacuum grease, and were then rinsed with Dulbecco's modified Eagle's medium (DMEM; Gibco BRL, Great Island, NY).

\section{Substrate analysis}

Protein-micropatterned bilayer surfaces were examined using established fluorescence microscopy techniques. Briefly, micropatterned surfaces were incubated with $5 \%$ BSA in PB for $1 \mathrm{~h}$, then incubated, at $37^{\circ} \mathrm{C}$ for $1 \mathrm{~h}$ each, with, first, an antibody to fibronectin (diluted 1:200 in PB; Sigma) generated in rabbit, and then with a Texas Red ${ }^{\circledR}$-labeled antibody to rabbit IgG (Molecular Probes). These fluorescently labeled samples were imaged using a Photometics (Photometrics, Ltd., Tucson, AZ) camera attached to a Nikon Eclipse microscope (Nikon Inc., Melville, NY).

Fluorescence recovery after photobleaching (FRAP) was used to demonstrate the fluidity of egg PC/NBD-PE lipid bilayers. ${ }^{16,17,24}$ On surfaces containing arrays of lipid corrals, an octagonal pattern was photobleached onto the prepared bilayer. Fluidity of the lipid bilayers was evidenced by the establishment of a uniform fluorescence within each corral over time; the final intensity in each corral reflects the fraction of fluorescently labeled lipids that were photobleached. Lipid diffusion was measured quantitatively by photobleaching a linear edge onto unpatterned lipid bilayers of egg PC/NBD-PE, and analyzing the time evolution of the fluorescence profile of this edge using a custom software package. Membrane fluidity was also examined by incorporating a small mol fraction of a fluorescent, negatively charged phospholipid, TR-PE, into supported bilayers. An electric field of $60 \mathrm{~V} / \mathrm{cm}$ was applied through the media (water) bathing this substrate, parallel to the membrane surface. ${ }^{16}$ Membrane fluidity was determined by observing whether the negatively charged TR-PE migrated in response to this applied field.

\section{Micropattern geometry}

Two micropattern geometries were examined in this report. Both geometries were described by a regular array of squares, each measuring either $5,10,20$, or $40 \mu \mathrm{m}$ in width, with each square being separated from its nearest neighbors by a distance of either $5,10,15,20$, or $30 \mu \mathrm{m}$. In one geometry, the squares corresponded to features of fibronectin, surrounded and separated by regions of lipid bilayer. Conversely, the second geometry consisted of a grid-like layout of fibronectin lines, surrounding and separating square corrals of lipid bilayer.

\section{Cell culture}

Cow pulmonary arterial endothelial cells (CPAE cells; CLL-209; American Tissue Culture Collection) were cultured in DMEM supplemented with $20 \%$ fetal bovine serum under standard cell culture conditions (a humidified, 5\% $\mathrm{CO}_{2} / 95 \%$ air environment maintained at $37^{\circ} \mathrm{C}$ ). These cells were routinely passaged to maintain logarithmic growth and were of passage 18 to 25 when used in cell experiments.

All cell adhesion experiments were carried out under serum-free conditions to minimize the effects of exogenous serum protein components. Subconfluent cultures of CPAE cells were dissociated using $0.25 \%$ trypsin in calcium- and magnesium-free DMEM, pelleted by centrifugation, and resuspended in fresh DMEM supplemented with $10 \mu \mathrm{g} / \mathrm{mL}$ of Cell Tracker Blue (Molecular Probes). These cells were plated onto prepared substrates at an areal density of $1.1 \times$ $10^{4}$ cells $/ \mathrm{cm}^{2}$, and then allowed to adhere for $6 \mathrm{~h}$ under standard cell culture conditions. Adherent cells were then fixed with cold $\left(4^{\circ} \mathrm{C}\right) 4 \%$ paraformaldehyde in calcium- and magnesium-free HEPES-buffered saline for $10 \mathrm{~min}$.

Adherent cells were then visualized by epifluorescence microscopy. Appropriate filter sets were used to separately visualize the Cell Tracker Blue, NBD, and Texas Red ${ }^{\circledR}$ fluorophores. Cell adhesion density on each unpatterned, reference substrate was calculated from the number of cells counted in five randomly selected fields, each measuring 3.9 $\mathrm{mm}^{2}$ in area. On all surfaces, cell morphology and, if appropriate, the location of lipid bilayers were recorded and categorized. Replicate experiments on all substrates were carried out three to five different times.

\section{RESULTS}

\section{Supported lipid bilayers inhibit endothelial cell adhesion}

Within $6 \mathrm{~h}$ after seeding, pulmonary endothelial cells adhere to and spread upon reference substrates 
of plain, unmodified glass [Fig. 2(A)]. This adhesion was observed in the presence of only trace amounts of adhesive proteins (i.e., under serum-free culture conditions), demonstrating the strongly adhesive nature of these cells. In contrast, endothelial cells seeded onto glass-supported bilayers of egg PC were round in morphology [Fig. 2(B)], and cell adhesion density was reduced by $85 \%$ compared to those on plain glass. This minimal adhesion of endothelial cells onto fluid lipid bilayers is in agreement with earlier reports that supported lipid structures inhibit fibroblast adhesion. ${ }^{12}$

Preincubation of the supported bilayer with BSA further reduced cell adhesion by $90 \%$, compared to that on lipid bilayers not exposed to BSA [Fig. 2(C)]. This reduction of cell adhesion by BSA incubation or "passivation" was not a result of disruption of the supported membrane by the adsorbed proteins. BSApassivated bilayers of egg PC/NBD-PE were uniform, and visually identical to bilayers that were not exposed to BSA (data not shown). In addition, the diffusion coefficient of NBD-PE lipids in bilayers of egg PC/NBD-PE was not altered by BSA passivation [1.9 \pm $0.9 \mu^{2} / \mathrm{s}$ vs. $1.3 \pm 0.5 \mu \mathrm{m}^{2} / \mathrm{s}$ (mean $\pm \mathrm{SD}, n=3$ ) for
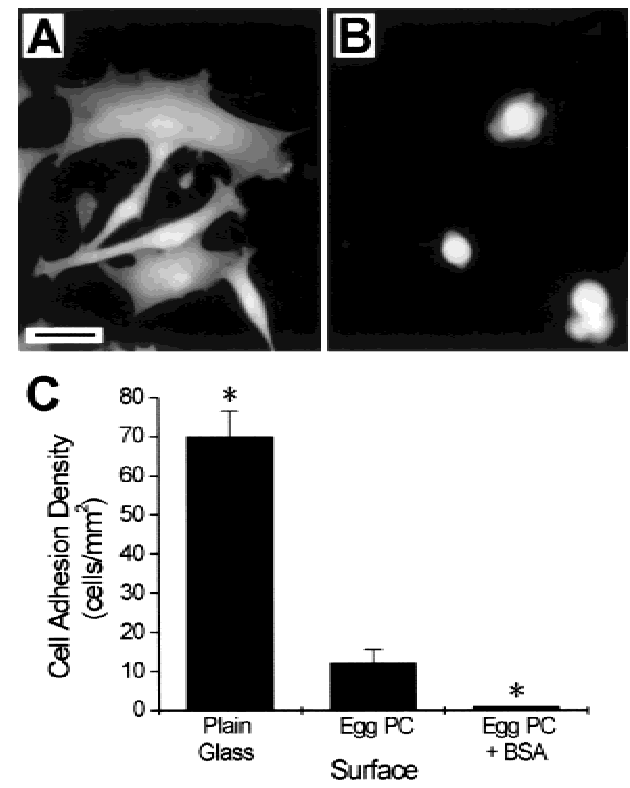

Figure 2. Fluid lipid bilayers do not support endothelial cell adhesion. (A) After $6 \mathrm{~h}$ in serum-free media, endothelial cells on substrates of plain glass exhibited a well-spread morphology. (B) In contrast, cells on surfaces supporting a fluid lipid bilayer of egg phosphatidylcholine (egg PC) exhibited a rounded morphology. (C) Cell adhesion density was reduced on lipid bilayers compared to plain glass. Passivation of the supported bilayers with $10 \mathrm{mg} / \mathrm{mL}$ of bovine serum albumin further reduced cell adhesion [egg PC + BSA, panel (C)]. Cells on egg PC + BSA surfaces resembled those on egg PC alone (B). The data in panel (C) are mean \pm SEM, $n=3 .{ }^{*} p<0.005$ (least significant difference test) compared to the substrate of egg PC. Panels (A) and (B) are presented at identical magnification; the scale bar in panel (A) $=25 \mu \mathrm{m}$. untreated and BSA passivated bilayers, respectively; these data are similar based on one-way ANOVA analysis with a criteria of $p<0.05]$. These results suggest that the reduced cell adhesion observed on BSApassivated lipid bilayers is mediated by an alternative mechanism, such as filling in of defects that are present in supported bilayer membranes. ${ }^{25-28}$ This may provide a useful method for measuring the characteristics of defects.

\section{Protein barriers direct lateral organization and diffusion of lipid bilayers}

Microcontact printing produced sharply defined barriers of fibronectin on glass substrates. Figure 1(B) illustrates one such pattern, a grid of fibronectin lines, each measuring $5 \mu \mathrm{m}$ in width and spaced $40 \mu \mathrm{m}$ apart. Upon subsequent incubation of this patterned surface with small unilamellar vesicles, lipid bilayers selectively assembled onto regions of plain glass, avoiding the protein-coated regions of the surface and forming an array of corralled lipid bilayers [Fig. 1(B)]. As shown in Figure 1(C), an octagonal pattern was photobleached onto the micropatterned surface, resulting in the photodestruction of different fractions of the fluorescently labeled lipid in adjacent corrals. After $10 \mathrm{~min}$, a uniform level of fluorescence was observed within each corral [Fig. 1(D)], indicating that the lipids in these bilayers diffused freely and mixed. Adjacent squares exhibited different levels of fluorescence, reflecting the area fraction irradiated in panel (C), demonstrating that the individual corrals are isolated from each other. These patterns were stable for several days, and did not degrade over the entire duration of storage or upon interaction with cells (see below).

\section{Fibronectin barriers promote endothelial cell adhesion onto lipid bilayers}

Adhesion of endothelial cells onto surfaces containing two complementary patterns of fibronectin and lipid bilayers was examined under serum-free cell culture conditions to minimize the influence of exogenous proteins. On all of these surfaces, endothelial cells selectively adhered to the patterns of fibronectin. The morphology of adherent cells was correlated with the geometry and dimensions of the fibronectin barriers. On surfaces patterned with squares of fibronectin surrounded and separated by bilayers of egg PC/ NBD-PE, adherent cells exhibited five general morphologies, which were categorized as " +2, , " +1 ," " -1, " " -2, " and "CS," reflecting the degree of cell 
spreading; the predominant morphology observed on each pattern is presented in Table I. On surfaces modified with large, closely spaced squares of fibronectin, adherent cells were well spread [Fig. 3(A)] and resembled cells on unpatterned, adhesive surfaces such as plain glass [Fig. 2(A)]; this well-spread morphology was categorized as +2 . Adherent cells spanned multiple features of fibronectin, bridging over the narrow, intervening regions of supported lipid bilayer. Decreasing the width of each fibronectin square and/or increasing the spacing between squares resulted in a reduction in cell spreading; adherent cells exhibited a branched morphology, as illustrated in Figure 3(B). This morphology was categorized as +1 . As was observed for the +2 morphology, cellular processes spanned multiple fibronectin squares, juxtaposing regions of the adherent cells to the lipid bilayers. Further reduction of the width of each square and/or increase of the spacing between squares resulted in continued reduction in cell spreading. Adherent cells exhibited the bipolar cell morphology illustrated in Figure 3(C), which was categorized as -1 . On these surfaces, adherent cells extended only thin processes across the intervening lipid bilayer regions. On surfaces patterned with the smallest fibronectin squares (which measured $5 \mu \mathrm{m}$ in width), adherent cells were rounded, occasionally elaborating thin processes that extended to a neighboring square. This morphology is illustrated in Figure 3(D), and was categorized as -2 . For these four classes of cell morphology, the best predictor of cell spreading was the fraction of surface occupied by lipid bilayer, a parameter referred to as

TABLE I

Cell Morphology as a Function of Pattern Dimensions

\begin{tabular}{|c|c|c|c|c|c|}
\hline & \multicolumn{4}{|c|}{ Width of Squares $(\mu \mathrm{m})$} \\
\hline & & 40 & 20 & 10 & 5 \\
\hline \multirow{5}{*}{ 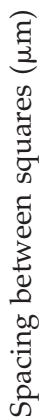 } & 5 & $+2(0.21)$ & $+2(0.36)$ & $+2(0.56)$ & \\
\hline & 10 & $+2(0.36)$ & $+2(0.56)$ & $+1(0.75)$ & $-2(0.89)$ \\
\hline & 15 & & $+2,+1(0.67)$ & $-1(0.84)$ & $-2(0.94)$ \\
\hline & 20 & CS (0.56) & $+1(0.75)$ & $-1(0.89)$ & $-2(0.96)$ \\
\hline & 30 & & $-2(0.84)$ & $-2(0.94)$ & $-2(0.98)$ \\
\hline
\end{tabular}

Six-h adhesion of endothelial cells onto surfaces patterned with squares of fibronectin. The predominant cell morphology (illustrated in Fig. 3) observed on each pattern is presented as a function of the width of and spacing between the fibronectin squares. The fraction of each surface occupied by lipid bilayer is indicated in parentheses. The inclusion of two symbols for $20-\mu \mathrm{m}$ wide squares spaced $15 \mu \mathrm{m}$ apart reflects the observation of large populations of both morphologies. the "bilayer coverage fraction," which is reported in Table I for each surface. Specifically, the transition from the +2 morphology to $+1,-1$, and finally -2 , which represents a decrease in cell spreading, was directly correlated to the bilayer coverage fraction, independent of the width or spacing of the fibronectin squares (Table I).

The final morphology observed on surfaces modified with squares of fibronectin is illustrated in Figure $3(\mathrm{E})$, and is characterized by adhesion and spreading of cells on individual features of fibronectin. Adherent cells did not extend processes across intervening regions of lipid bilayer. This confined spreading (CS) morphology was observed on fibronectin squares measuring $40 \mu \mathrm{m}$ in width and spaced $20 \mu \mathrm{m}$ or greater from neighboring squares.

On surfaces patterned with grids of fibronectin surrounding corrals of lipid bilayers, adherent cells exhibited two morphologies. Cells adherent to surfaces patterned with lipid corrals measuring either 10 or 20 $\mu \mathrm{m}$ in width exhibited a well-spread morphology, covering either large fractions or entire areas of multiple corrals; this morphology is illustrated in Figure 4(A), which shows cells on a surface containing an array of $20-\mu \mathrm{m}$ wide corrals. In contrast, cells on surfaces containing lipid corrals measuring $40 \mu \mathrm{m}$ in width extended multiple, long processes selectively along the fibronectin grids [Fig. 4(B)]. Cell bodies also selectively adhered to fibronectin, but rarely extended across the lipid corrals. Cell morphology on these surfaces was only correlated with the width of the lipid bilayer corrals and was independent of the width of the fibronectin gridlines.

Cell adhesion over a region of lipid bilayer did not influence the fluid properties of the supported membrane. Figure 5(A) illustrates 6-h adhesion of endothelial cells onto a surface containing an array of $20-\mu \mathrm{m}$ wide lipid corrals (surrounded by fibronectin) composed of egg PC/TR-PE. After the cells were fixed, an electric field of $60 \mathrm{~V} / \mathrm{cm}$ was applied parallel to the membrane surface, inducing migration of the negatively charged, fluorescently labeled TR-PE lipids to the right side of each corral [Fig. 5(B)]. Gradients were formed in corrals underlying an adherent cell that were identical to corrals not covered by a cell, indicating that the mobility of lipids in these supported membranes was not affected by cell adhesion over the bilayer regions. This lateral mobility of lipids under adherent cells suggests that the distance between the cell surface and the supported membrane is greater than about $10 \AA$, the likely extent of the dye headgroup of TR-PE above the membrane surface. In preliminary experiments we have found that $40-\mathrm{nm}$ diameter beads attached to the headgroups of lipids in supported bilayers do not diffuse under adherent cells, suggesting an upper limit to the distance between the cell surface and supported membrane. This approach 

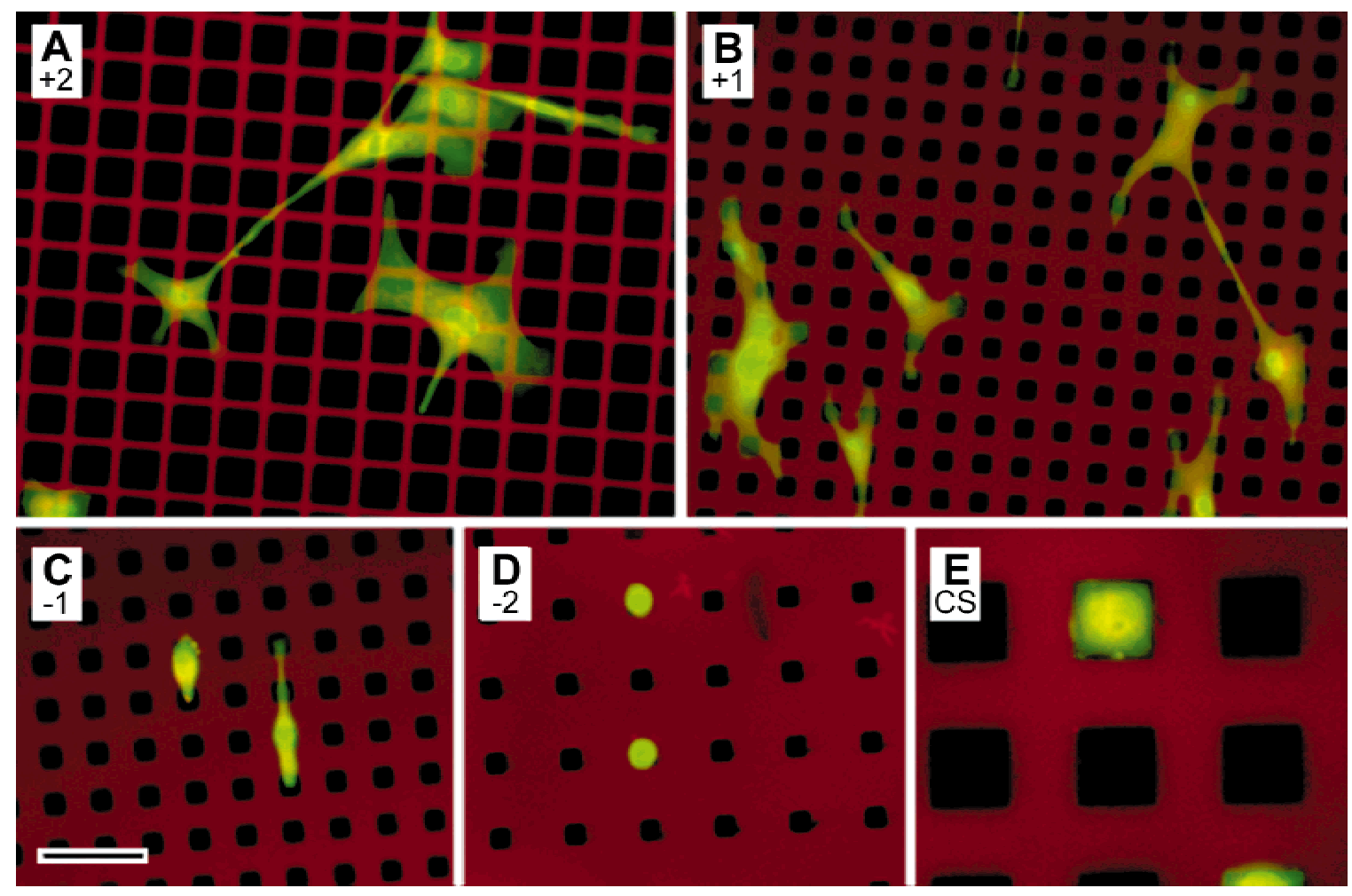

Figure 3. Adhesion of endothelial cells onto surfaces modified with squares of fibronectin. Representative epifluorescence images illustrating 6-h adhesion of endothelial cells, under serum-free conditions, onto surfaces patterned with square features of fibronectin (dark regions) surrounded by supported bilayers comprised of egg PC/NBD-PE (shown in gray here and in red in the online color image). Cells were labeled with Cell Tracker Blue and are shown in light gray (green, for clarity in the color images). Five categories of cell morphology, which reflect the degree of cell spreading, were observed on these surfaces. A symbol for each category is included next to the appropriate panel label and corresponds to the symbols reported in Table I. The width and spacing of squares in each frame are as follows: (A) $20 \mu \mathrm{m}$ squares spaced $5 \mu \mathrm{m}$ apart; (B) $10 \mu \mathrm{m}$ squares spaced $10 \mu \mathrm{m}$ apart; (C) $10 \mu \mathrm{m}$ squares spaced $15 \mu \mathrm{m}$ apart; (D) $10 \mu \mathrm{m}$ squares spaced $30 \mu \mathrm{m}$ apart; and (E) $40 \mu \mathrm{m}$ squares spaced $30 \mu \mathrm{m}$ apart. All images are presented at identical magnification; the scale bar in panel $(\mathrm{C})=50 \mu \mathrm{m}$. [Color figure can be viewed in the online issue, which is available at www.interscience.wiley.com.]

to measuring the vertical distance between the cell and supported membrane surfaces relies on the lateral fluidity of the underlying supported membrane and will be reported in detail in a subsequent communication.

\section{DISCUSSION}

Chemical micropatterning has provided a powerful technique for controlling cellular response to material surfaces. ${ }^{19,21,29-36}$ These studies have focused primarily on the interaction between cells and cell-adhesive compounds patterned and immobilized on a substrate; the complementary regions are often considered to be inert, nonadhesive substrates. In contrast, the goal of the present study is to use micropatterning to promote the juxtaposition of cells with a biologically important but inherently nonadhesive surface, a supported lipid bilayer. By demonstrating that micropatterned anchorages of fibronectin can facilitate the adhesion and spreading of endothelial cells across a substrate containing a supported membrane, this study establishes a new application of chemical micropatterning for controlling cell interactions with biomaterial surfaces.

Both square and grid-like patterns of fibronectin were effective in promoting cell adhesion onto lipid bilayer surfaces. However, cell response to these two protein-micropatterned surfaces was qualitatively different. On surfaces patterned with squares of fibronectin, maximum cell spreading was observed on large, closely spaced fibronectin features [Fig. 3(A) and Table I]. Because the majority of the surface was coated with fibronectin, however, only a small fraction of each cell was exposed to the lipid bilayer. Increasing the bilayer coverage fraction could promote greater overlap between an adherent cell and lipid bilayer. However, increasing the fraction of the surface covered with lipid bilayer reduced cell spreading (Fig. 3 and Table I), decreasing the interaction between the adherent cell and the supported membrane; on surfaces containing greater than 75 areal percent of lipid bilayer, adherent cells were either bipolar or 


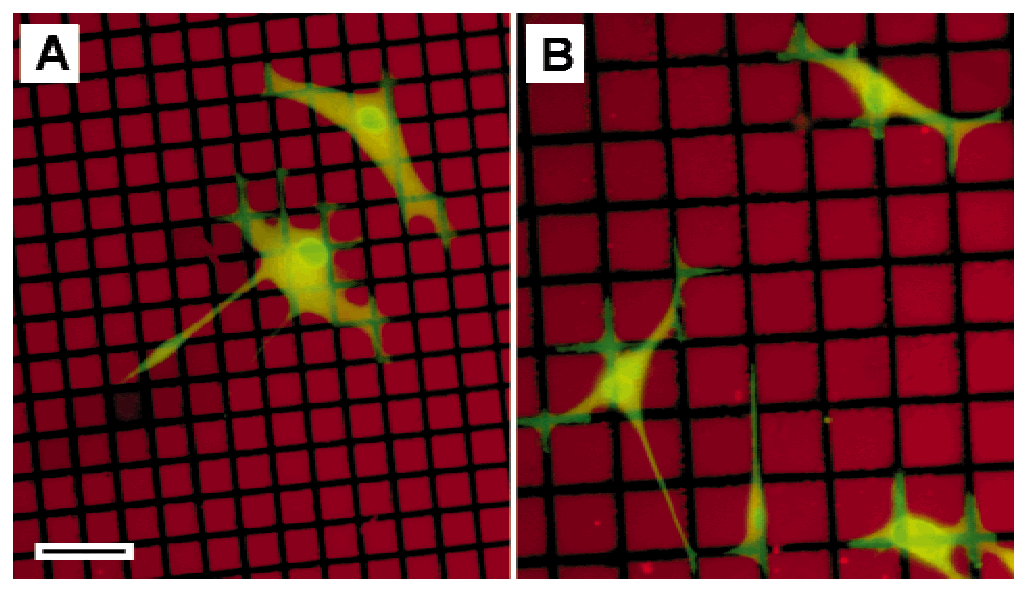

Figure 4. Adhesion of endothelial cells onto surfaces modified with grids of fibronectin. Endothelial cell (light gray, or green) adhesion onto surfaces patterned with grid-like features of fibronectin (dark horizontal and vertical lines) corralling supported bilayers of egg PC/NBD-PE (shown in gray, or red). Cell morphology was correlated with the dimensions of the lipid corrals, independent of the width of the fibronectin grid lines. These representative images illustrate endothelial cell morphology on surfaces containing lipid corrals of width (A) $20 \mu \mathrm{m}$ (separated by $5 \mu \mathrm{m}$ ) and (B) $40 \mu \mathrm{m}$ (separated by $10 \mu \mathrm{m}$ ). Both images are presented at identical magnification; the scale bar in panel $(A)=50 \mu \mathrm{m}$. [Color figure can be viewed in the online issue, which is available at www.interscience.wiley.com.]
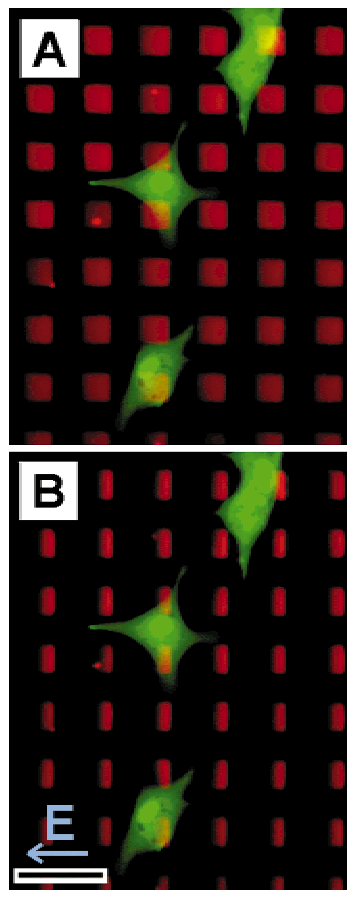

Figure 5. Lipid bilayers underlying adherent cells remain fluid. (A) Six-h adhesion of endothelial cells (shown in light gray, or green) on a surface containing $20-\mu \mathrm{m}$ wide corrals of egg PC/TR-PE (shown as square regions of gray, or red). (B) After $5 \mathrm{~min}$ of exposure to a $60 \mathrm{~V} / \mathrm{cm}$ electric field applied parallel to the membrane surface, the negatively charged TR-PE lipids underlying adherent cells migrated to the right side of each corral in a manner identical to those in regions distant from the cells. The scale bar in panel $(B)=50$ $\mu \mathrm{m}$. [Color figure can be viewed in the online issue, which is available at www.interscience.wiley.com.] rounded. Reduction of cell spreading has also been associated with a decrease in cell survival. ${ }^{19}$ In contrast, increasing the bilayer coverage fraction on surfaces containing grids of fibronectin by decreasing the gridline width does not decrease cell spreading; cell spreading on these surfaces was purely a function of the spacing between gridlines (Fig. 4). Surfaces containing corrals of lipid bilayers of either 10 or $20 \mu \mathrm{m}$ width are of particular interest, as adherent cells were able to completely cover individual lipid corrals [Fig. $4(\mathrm{~A})]$.

The two geometries of micropatterned protein barriers offer different, complementary advantages for studying cell-membrane interaction. On surfaces containing squares of fibronectin, the complementary regions of lipid bilayer form a single, connected membrane. These canals of fluid lipid bilayer could be used to introduce membrane-associated biomolecules into the interface between an adherent cell and the substrate, for example by application of an electric field, as we have shown in a different context. ${ }^{13}$ By comparison, surfaces modified with grids of fibronectin contain multiple, isolated corrals of lipid. Using recently developed methods for controlling the composition of individual bilayer patches, ${ }^{17,37-39}$ these arrays of lipid corrals should make possible the quantitative, concentration-dependent study of cell response to membrane-associated biomolecules. Lastly, by combining canals and corrals of lipids delineated by fibronectin, or other extracellular matrix proteins, we can take a step towards mimicking aspects of the environment encountered by populations of cells organized in tissues.

In summary, protein-micropatterned lipid bilayers 
introduce a new level of sophistication into investigating how membrane-associated biomolecules, which can be incorporated into the synthetic supported bilayers, mediate cellular function. These surfaces potentially facilitate the study of how anchoragedependent cells recognize and respond to membrane biomolecules, free of the complexity introduced by the presence of other membrane components or internal cellular structures. Reorganization of either endogenous or engineered ${ }^{40}$ molecular species incorporated into membranes of adherent cells using electric fields, a concept that has already proven useful in several contexts, ${ }^{41,42}$ may provide additional insight into the mechanisms that regulate cell-membrane interactions. Finally, we can speculate that incorporation of cellcell communication proteins, such as gap junctions, and electronics integrated into the solid support, could be used to probe the internal state of a cell, leading to advanced, cell-based devices.

The authors would like to thank Steven S. Andrews and Caroline M. Ajo for the fitting program and algorithm used to extract diffusion coefficients from electrophoresis data, and Professor Chaitan Khosla for providing the cell culture facilities utilized for this work. The Stanford Nanofabrication Facility (SNF) is gratefully acknowledged for support in fabrication.

\section{References}

1. Bayerl TM, Bloom M. Physical-properties of single phospholipid-bilayers adsorbed to micro glass-beads : a new vesicular model system studied by $2 \mathrm{H}$ nuclear magnetic-resonance. Biophys J 1990;58:357-362.

2. Johnson SJ, Bayerl TM, McDermott DC, Adam GW, Rennie AR, Thomas RK, Sackmann E. Structure of an adsorbed dimyristoylphosphatidylcholine bilayer measured with specular reflection of neutrons. Biophys J 1991;59:289-294.

3. Koenig BW, Kruger S, Orts WJ, Majkrzak CF, Berk NF, Silverton JV, Gawrisch K. Neutron reflectivity and atomic force microscopy studies of a lipid bilayer in water adsorbed to the surface of a silicon single crystal. Langmuir 1996;12:1343-1350.

4. Schoenwaelder SM, Burridge K. Bidirectional signaling between the cytoskeleton and integrins. Curr Opinion Cell Biol 1999;11:274-286.

5. Giancotti FG, Ruoslahti E. Transduction : integrin signaling. Science 1999;285:1028-1032.

6. Viola A, Lanzavecchia A. T-cell activation and the dynamic world of rafts. Apmis 1999;107:615-623.

7. Sackmann E. Supported membranes: scientific and practical applications. Science 1996;271:43-48.

8. Watts TH, McConnell HM. Biophysical aspects of antigen recognition by T cells. Ann Rev Immunol 1987;5:461-475.

9. McConnell HM, Watts TH, Weis RM, Brian AA. Supported planar membranes in studies of cell-cell recognition in the immune system. Biochim Biophys Acta 1986;864:95-106.

10. Grakoui A, Bromley SK, Sumen C, Davis MM, Shaw AS, Allen PM, Dustin ML. The immunological synapse: a molecular machine controlling $\mathrm{T}$ cell activation. Science 1999;285:221-227.

11. Dori Y, Bianco-Peled H, Satija SK, Fields GB, McCarthy JB, Tirrell M. Ligand accessibility as means to control cell response to bioactive bilayer membranes. J Biomed Mater Res 2000;50: 75-81.

12. Margolis LB, Dyatlovitskaya EV, Bergelson LD. Cell-lipid interactions. Cell attachment to lipid substrates. Exp Cell Res 1978;111:454-457.

13. van Oudenaarden A, Boxer SG. Brownian ratchets: molecular separations in lipid bilayers supported on patterned arrays. Science 1999;285:1046-1048.

14. Groves JT, Ulman N, Boxer SG. Micropatterning of fluid lipid bilayers on solid supports. Science 1997;275:651-653.

15. Cremer PS, Groves JT, Kung LA, Boxer SG. Writing and erasing barriers to lateral mobility into fluid phospholipid bilayers. Langmuir 1999;15:3893-3896.

16. Groves JT, Boxer SG. Electric field-induced concentration gradients in planar supported bilayers. Biophys J 1995;69:19721975.

17. Hovis JS, Boxer SG. Patterning barriers to lateral diffusion in supported lipid bilayer membranes by blotting and stamping. Langmuir 2000;16:894-897.

18. Kumar A, Biebuyck HA, Whitesides GM. Patterning selfassembled monolayers: applications in material science. Langmuir 1994;10:1498-1511.

19. Chen CS, Mrksich M, Huang S, Whitesides GM, Ingber DE. Geometric control of cell life and death. Science 1997;276:14251428.

20. James CD, Davis RC, Kam L, Craighead HG, Isaacson M, Turner JN, Shain W. Patterned protein layers on solid substrates by thin stamp microcontact printing. Langmuir 1998;14: 741-744.

21. Kam L, James CD, Withers G, Banker G, Craighead HG, Isaacson M, Turner JN, Shain W. Neuron attachment and outgrowth on microcontact-printed polylysine-conjugated laminin. J Neurosci Meth 2000, in press.

22. Kung LA, Kam L, Hovis JS, Boxer SG. Patterning hybrid surfaces of proteins and supported lipid bilayers. Langmuir 2000; 16:6773-6776.

23. Brian AA, McConnell HM. Allogeneic stimulation of cytotoxic $\mathrm{T}$ cells by supported planar membranes. Proc Nat Acad Sci USA 1984;81:6159-6163.

24. Stelzle M, Miehlich R, Sackmann E. 2-Dimensional microelectrophoresis in supported lipid bilayers. Biophys J 1992;63: 1346-1354.

25. Hui SW, Viswanathan R, Zasadzinski JA, Israelachvili JN. The structure and stability of phospholipid bilayers by atomic force microscopy. Biophys J 1995;68:171-178.

26. Mou J, Yang J, Shao Z. Tris(hydroxymethyl)aminomethane $(\mathrm{C} 4 \mathrm{H} 11 \mathrm{NO3})$ induced a ripple phase in supported unilamellar phospholipid bilayers. Biochem 1994;33:4439-4443.

27. Mou J, Yang J, Shao Z. Atomic force microscopy of cholera toxin B-oligomers bound to bilayers of biologically relevant lipids. J Mol Biol 1995;248:507-512.

28. Radler J, Radmacher M, Gaub HE. Velocity-dependent forces in atomic-force microscopy imaging of lipid films. Langmuir 1994;10:3111-3115.

29. Bhatia SN, Yarmush ML, Toner M. Controlling cell interactions by micropatterning in co-cultures: hepatocytes and $3 \mathrm{~T} 3$ fibroblasts. J Biomed Mater Res 1997;34:189-199.

30. Healy KE, Thomas CH, Rezania A, Kim JE, McKeown PJ, Lom $\mathrm{B}$, Hockberger PE. Kinetics of bone cell organization and mineralization on materials with patterned surface chemistry. Biomaterials 1996;17:195-208.

31. James CD, Davis R, Meyer M, Turner A, Turner S, Withers G, Kam L, Banker G, Craighead H, Isaacson M, Turner J, Shain W. Aligned microcontact printing of micrometer-scale poly-Llysine structures for controlled growth of cultured neurons on planar microelectrode arrays. IEEE Trans Biomed Eng 2000;47: 17-21.

32. Kam L. Modulation of neuron and astroglial cell function by 
micropatterning and immobilization of select biomolecules on biomaterial surfaces. Troy, NY: Dep Biomed Eng Rensselaer Polytechnic Institute; 1999. p 205.

33. Kleinfeld D, Kahler KH, Hockberger PE. Controlled outgrowth of dissociated neurons on patterned substrates. J Neurosci 1988;8:4098-4120.

34. Saneinejad S, Shoichet MS. Patterned glass surfaces direct cell adhesion and process outgrowth of primary neurons of the central nervous system. J Biomed Mater Res 1998;42:13-19.

35. St. John PM, Kam L, Turner SW, Craighead HG, Isaacson M, Turner JN, Shain W. Preferential glial cell attachment to microcontact-printed surfaces. J Neurosci Meth 1997;75:171-177.

36. Singhvi R, Kumar A, Lopez GP, Stephanopoulos GN, Wang DIC, Whitesides GM, Ingber DE. Engineering cell shape and function. Science 1994;264:696-698.

37. Cremer PS, Yang T. Creating spatially addressed arrays of pla- nar supported fluid phospholipid membranes. J Am Chem Soc 1999;121:8130-8131.

38. Kung LA, Groves JT, Ulman N, Boxer SG. Printing via photolithography on micropartitioned fluid lipid membranes. Adv Mat 2000;12:731-734.

39. Kam L, Boxer SG. Formation of supported lipid bilayer composition arrays by controlled mixing and surface capture. J Am Chem Soc, to appear.

40. Saxon E, Bertozzi CR. Cell surface engineering by a modified Staudinger reaction. Science 2000;287:2007-2010.

41. Lin-Liu S, Adey WR, Poo MM. Migration of cell surface concanavalin A receptors in pulsed electric fields. Biophys J 1984; 45:1211-1217.

42. Webb WW, Barak LS, Tank DW, Wu ES. Molecular mobility on the cell surface. Biochem Soc Symp 1981;46:191-205. 